\title{
Análisis de la demanda asistencial neurológica generada por la atención primaria en una área geográfica de las comarcas de Girona
}

\author{
S. López-Pousa ${ }^{\text {a,b }}$, S. Monserrat-Vila ${ }^{\text {b }}$, O. Turró-Garriga ${ }^{\text {b }}$, M. Aguilar-Barberà ${ }^{c}$, \\ C. Caja-López $^{\text {c }}$, J. Vilalta-Franch ${ }^{\text {a,b }}$, I. Pericot-Nierga ${ }^{a}$, A. Turon-Estrada ${ }^{\text {a }}$, \\ J. Turbau-Recio ${ }^{a}$, G. Coll-De Tuero ${ }^{b}$, J. Garre-Olmo ${ }^{b}$
}

\author{
ANÁLISIS DE LA DEMANDA ASISTENCIAL NEUROLÓGICA GENERADA POR LA \\ ATENCIÓN PRIMARIA EN UNA ÁREA GEOGRÁFICA DE LAS COMARCAS DE GIRONA
}

\begin{abstract}
Resumen. Objetivo. Análisis descriptivo de la actividad ambulatoria en un servicio de neurología en relación con la frecuencia y tipo de enfermedades neurológicas atendidas. Pacientes y métodos. Estudio retrospectivo y trasversal de los pacientes remitidos a la consulta ambulatoria de neurología. Se registraron consecutivamente los casos visitados por primera vez durante los años 2006 y 2007. Se evaluó la documentación médica a partir de los registros hospitalarios informatizados, recogiéndose: zona de salud de referencia, fecha de visita, edad, sexo y diagnóstico según la Clasificación Internacional de Enfermedades, 9. ${ }^{a}$ edición, modificación clínica (CIE-9-MC), reconvertida a la Clasificación Internacional de Enfermedades, 10. ${ }^{a}$ edición (CIE-10). Resultados. La media de edad fue de 60,6 \pm 20,9 años (rango: 4-95 años) y el 61,4\% fueron mujeres. El índice de frecuentación fue de 4,3 solicitudes/1.000 habitantes para el año 2006 y de 3,9 para el año 2007, y se observó un incremento con la edad para las enfermedades neurodegenerativas. Respecto a la CIE-10, las enfermedades se clasificaron como episódicas y paroxísticas (25\%), degenerativas y desmielinizantes (18,6\%), trastornos mentales orgánicos (14,7\%), síndromes extrapiramidales (10,5\%), enfermedades de la circulación cerebral (3,5\%), trastornos relacionados con el estrés y somatomorfos (3,5\%) y enfermedades del oído interno (3,3\%). Las demás patologías no alcanzaron el 3\%. El 61\% de los pacientes presentó enfermedades del sistema nervioso central y el 20,3\%, trastornos psiquiátricos. Las enfermedades más frecuentes fueron los trastornos cognitivos (31,5\%), las cefaleas (18,6\%) y los trastornos del movimiento (11,7\%), seguidas de las enfermedades psiquiátricas, epilepsias, enfermedades cerebrovasculares y neuropatías. Conclusiones. La frecuentación de las visitas aumenta con la edad, y el orden según la frecuencia fue: episódicas y paroxísticas, degenerativas y desmielinizantes, trastornos psicóticos y del comportamiento, y síndromes extrapiramidales. [REV NEUROL 2009; 49: 288-94]
\end{abstract}

Palabras clave. Atención primaria. Demanda neurológica. Diagnóstico. Enfermedades neurológicas. Epidemiología.

\section{INTRODUCCIÓN}

Desde la segunda mitad del siglo Xx se ha producido un progresivo envejecimiento de la población, tanto en los países desarrollados como en los países en vías de desarrollo [1]. Este envejecimiento poblacional ha favorecido un incremento de las enfermedades neurodegenerativas. Se calcula que entre un 6-15\% de la población actual presenta una enfermedad neurológica [2,3], y que son estas enfermedades las que provocan mayor discapacidad y mortalidad [4].

En nuestro país, las enfermedades neurológicas han aumentado considerablemente, sobre todo aquellas relacionadas con la edad, debido, sobre todo, al incremento de la esperanza de vida, a los avances diagnósticos y terapéuticos, y a una mejor educación sanitaria [5,6]. En este sentido, la prevalencia de demencia en población de 70 y más años se estima entre un 9,4\% [7] y un

Aceptado tras revisión externa: 29.06.09.

${ }^{a}$ Servicio de Neurociencias Clínicas. ${ }^{b}$ Unidad de Investigación. Institut d'Assistència Sanitària. Salt, Girona. ${ }^{c}$ Plan Director Sociosanitario. Departamento de Salud. Generalitat de Catalunya. Barcelona, España.

Correspondencia: Dr. Secundino López-Pousa. Institut d'Assistència Sanitària. Doctor Castany, s/n. E-17190 Salt (Girona). Fax: +34 972189017. E-mail: uvamid@ias.scs.es

Estudio financiado por una beca del Plan Director Sociosanitario del Departamento de Salud de la Generalitat de Catalunya.

(C) 2009, REVISTA DE NEUROLOGÍA
$16,3 \%$ [8], y en la enfermedad de Parkinson (EP), del 0,9\% [9] en la población mayor de 40 años y del 1,5\% [10,11] en la población de 65 y más años. Otras patologías, como las cefaleas, las distrofias musculares y el síndrome vertiginoso, a pesar de su alta frecuencia, no han aumentado, ya que su presentación disminuye en las etapas tardías de la vida [12,13].

La Organización Mundial de la Salud, desde hace años, anima a que se realicen estudios sobre la disposición y utilización de los servicios de salud con la finalidad de conocer la morbilidad de las distintas patologías médicas [14]. En este sentido, en nuestro país se han realizado numerosos estudios descriptivos sobre la asistencia neurológica del sistema sanitario [5,15-45]. A pesar de estos estudios, actualmente no existen registros de base poblacional de las enfermedades neurológicas más frecuentes, ni tampoco de cómo se distribuyen en los diferentes dispositivos asistenciales, debido en gran parte a que no existe la obligación de utilizar la Clasificación Internacional de las Enfermedades, 9. ${ }^{a}$ edición, modificación clínica, y 10. ${ }^{\text {e edición, }}$ modificación clínica (CIE-9-MC/CIE-10-MC) [46,47], en las consultas externas de los centros hospitalarios o desde las consultas especializadas de los centros ambulatorios de las áreas básicas.

En todo el territorio español, todos los centros sanitarios públicos y privados registran en los pacientes ingresados, de modo obligatorio, un conjunto mínimo básico de datos en el que se recoge información sobre la patología atendida, con la finalidad 
Tabla I. Número de casos e índice de frecuentación estratificado por grupos de edad para los años 2006 y 2007.

\begin{tabular}{lcccc}
\hline & Casos & Población (media) & IF & IC 95\% \\
\hline 0-14 años & 21 & 42.075 & 0,50 & $0,27-0,72$ \\
\hline 14-19 años & 14 & 6.742 & 2,08 & $0,92-3,24$ \\
\hline 20-29 años & 46 & 20.194 & 2,25 & $1,6-2,96$ \\
\hline 30-39 años & 48 & 23.471 & 2,05 & $1,45-2,64$ \\
\hline $40-49$ años & 56 & 19.836 & 2,80 & $2,06-3,59$ \\
\hline $50-59$ años & 59 & 14.881 & 3,96 & $2,92-5,01$ \\
\hline $60-69$ años & 77 & 10.645 & 7,23 & $5,58-8,89$ \\
\hline 70-79 años & 132 & 9.658 & 13,62 & $11,3-16,03$ \\
\hline $80+$ años & 105 & 5.500 & 19,09 & $15,38-22,8$ \\
\hline Total b & 518 & 89.987 & 5,76 & $5,26-6,26$ \\
\hline
\end{tabular}

IC 95\%: intervalo de confianza al 95\%; IF: índice de frecuentación. ${ }^{a}$ Casos por 1.000 habitantes; ${ }^{b}$ En el cálculo del total no se ha tenido en cuenta la población pediátrica.

de disponer de un banco de datos exhaustivo y válido sobre la actividad y morbilidad sanitaria que, sin duda, es útil para la planificación y la evaluación de recursos y compra de servicios. Los tipos de variables que se recogen son: identificación del paciente, variables relacionadas con el proceso y variables clínicas (diagnóstico y procedimientos codificados de acuerdo con la CIM-9-MC) [48]. A pesar de la utilidad de estos registros, su ámbito de influencia es estrictamente hospitalario y no ofrece información relativa a la actividad hospitalaria ambulatoria.

El objetivo principal del presente trabajo fue realizar un análisis descriptivo de la frecuencia y del tipo de enfermedades neurológicas atendidas durante el bienio 2006-2007 en un servicio de neurología con actividad ambulatoria, remitidas por el médico especialista en medicina familiar y comunitaria. Asimismo, y como objetivos secundarios, se agruparon y describieron los grupos diagnósticos correspondientes a las solicitudes de primera visita, y se realizó una propuesta de agrupación.

\section{PACIENTES Y MÉTODOS \\ Diseño}

Registro consecutivo de los casos visitados por primera vez, entre el 1 de enero y el 31 de diciembre de los años 2006 y 2007, en la Unidad de Neurología (UN) del Servicio de Neurociencias Clínicas del Hospital Santa Caterina de Girona y la Unidad de Valoración de la Memoria y las Demencias (UVaMiD), que es la unidad de referencia para el diagnóstico y seguimiento de los casos de trastorno cognitivo de las comarcas de Girona.

\section{Situación geográfica y población de referencia}

La UN atiende a la población de referencia de siete áreas básicas de salud, de las cuales tres son gestionadas por el Institut d'Assistència Sanitària (Anglès, Cassà de la Selva y Breda-Hostalric) y cuatro son gestionadas por el Institut Català de la Salut (Arbúcies-St. Hilari, Salt, Santa Coloma de Farners y Sils/Vidreres/Maçanet). En total, estas áreas básicas de salud están formadas por un total de 32 municipios, con una población global de 130.267 habitantes para el año 2006 y 133.857 para el año 2007 .

La UVaMiD actualmente está adscrita a la UN como un equipo de evaluación integral ambulatoria en trastornos cognitivos y atendió a una población de referencia de 687.331 habitantes en el año 2006 y de 706.185 en 2007.
En el presente estudio solamente se ha tenido en cuenta la población de referencia que comparten la UN y la UVaMiD.

\section{Procedimiento}

La información se recogió a través del registro informático del hospital (SAP AG ${ }^{\odot}$; Systeme, Anwendungen und Produkte) de los pacientes visitados en la UN por primera vez durante el período de estudio.

Para cada paciente se recogió el área básica de salud de referencia, la fecha de la visita, la edad, el sexo, el diagnóstico principal según la CIE-9-MC [48], y una variable de texto libre que permite la versión utilizada en el centro del SAP, en la que el facultativo puede escribir el diagnóstico fuera de los códigos CIE-9-MC. Se realizó la conversión de los diagnósticos CIE-9-MC a CIE-10-MC de acuerdo con la variable diagnóstica de texto libre y/o tras revisar la historia clínica. Los diagnósticos se clasificaron en base a los criterios CIE-10, que establece 22 capítulos.

\section{Tratamiento estadístico}

El análisis estadístico se realizó a partir de las variables de estudio mediante técnicas de tendencia central y dispersión para las variables cuantitativas, y mediante frecuencias relativas y absolutas para las variables cualitativas. Se aplicaron técnicas de contraste de hipótesis bivariantes paramétricas y no paramétricas según la distribución de los datos para determinar la presencia de diferencias significativas entre los grupos.

Los resultados se expresan como números absolutos y porcentajes, medias, desviación estándar e intervalos de confianza (IC) al 95\%. El procesamiento y análisis de los datos se realizaron mediante el programa estadístico SPSS, v. 14.0 para Windows.

El índice de frecuentación (IF) se determinó como el número de solicitudes de primera visita realizadas por cada 1.000 habitantes/año. La población de referencia del Hospital Santa Caterina se determinó a partir de los datos de la revisión municipal del padrón municipal 2007 del Instituto Nacional de Estadística [49].

\section{RESULTADOS}

\section{Demanda asistencial}

La demanda asistencial de las primeras visitas fue de 1.078 visitas (554 el año 2006 y 524 el año 2007).

\section{Datos demográficos}

La distribución por grupos de edad fue muy similar entre los años 2006 (228 varones, edad media: $58,4 \pm 21,8$ años; y 326 mujeres, edad media: $60,7 \pm 20,8$ años) y 2007 (227 varones, edad media: 60,8 $\pm 19,4$ años; y 297 mujeres, edad media: 62,1 $\pm 21,4$ años), con una mayor frecuencia de casos en las décadas de 70-79 y 80 o más años. La media de edad de todos los pacientes fue de 60,6 $\pm 20,9$ años (rango: 4-95 años). Se observó un ligero incremento en la media de edad de los pacientes remitidos el año 2007. Globalmente, el $61,4 \%$ fueron mujeres.

\section{Índice de frecuentación}

El IF global para los años 2006 y 2007 fue de 4,10/1.000 habitantes/año para la población global: 4,3 (IC 95\% = 3,9-4,6) para 2006 y 3,9 (IC 95\%= 3,6-4,3) para 2007, y 5,8 para la población mayor de 15 años (Tabla I). El IF aumentó progresivamente en todos los grupos de edad, duplicándose en la década de los 50-60 y de los 60-70. En edades más avanzadas, la frecuentación fue más elevada (del 20,1 en 2006 y del 18,2 en 2007 en la población de 80 o más años). Por patologías y grupos de edad, se observó que en las enfermedades degenerativas, como enfermedad de Alzheimer (EA), deterioro cognitivo leve, EP, demencia vascular y demencia mixta, el IF se incrementó con la edad, sobre todo a partir de los 60 años, mientras que en otras patologías, como cefalea tensional, migraña y epilepsia, se mantuvo estable o con pequeñas diferencias (Tabla II).

\section{Patología atendida por grupos diagnósticos}

En base a los criterios de la CIE-10, las enfermedades o grupos diagnósticos se clasificaron como: episódicas y paroxísticas $(25,1 \%)$, degenerativas y desmielinizantes $(18,6 \%)$, trastornos mentales orgánicos $(14,7 \%)$, síndromes extrapiramidales $(10,5 \%)$, enfermedades de la circulación cerebral $(4,5 \%)$, trastornos relacionados con el estrés y somatomorfos $(3,5 \%)$ y enfermedades del oído interno (3,3\%). Las demás patologías no alcanzan el 3\% (Tabla 
Tabla II. Número de casos de las enfermedades más frecuentes e índice de frecuentación estratificado por grupos de edad agrupado para los años 2006 y 2007.

\begin{tabular}{|c|c|c|c|c|c|c|c|c|c|c|c|c|c|c|c|c|c|c|}
\hline & \multicolumn{2}{|c|}{$<10$ años } & \multicolumn{2}{|c|}{ 10-19 años } & \multicolumn{2}{|c|}{ 20-29 años } & \multicolumn{2}{|c|}{ 30-39 años } & \multicolumn{2}{|c|}{ 40-49 años } & \multicolumn{2}{|c|}{ 50-59 años } & \multicolumn{2}{|c|}{ 60-69 años } & \multicolumn{2}{|c|}{ 70-79 años } & \multicolumn{2}{|c|}{$80+$ años } \\
\hline & $n$ & IF & $n$ & $\mathrm{IF}$ & $n$ & $\mathrm{IF}$ & $n$ & IF & $n$ & IF & $n$ & $\mathrm{IF}$ & $n$ & IF & $n$ & $\mathrm{IF}$ & $n$ & IF \\
\hline Enfermedad de Alzheimer & 0 & 0 & 0 & 0 & 0 & 0 & 0 & 0 & 1 & 0,05 & 2 & 0,13 & 13 & 1,22 & 60 & 6,21 & 92 & 16,73 \\
\hline Cefalea tensional & 0 & 0 & 8 & 0,61 & 18 & 0,89 & 18 & 0,77 & 19 & 0,96 & 13 & 0,87 & 10 & 0,94 & 14 & 1,45 & 6 & 1,09 \\
\hline Deterioro cognitivo leve & 0 & 0 & 0 & 0 & 0 & 0 & 1 & 0,04 & 2 & 0,1 & 8 & 0,54 & 23 & 2,16 & 37 & 3,83 & 24 & 4,36 \\
\hline Migraña & 0 & 0 & 4 & 0,31 & 15 & 3,74 & 16 & 0,68 & 14 & 0,71 & 9 & 0,6 & 6 & 0,56 & 10 & 1,04 & 3 & 0,55 \\
\hline Neuropatías & 0 & 0 & 0 & 0 & 5 & 0,25 & 6 & 0,26 & 14 & 0,71 & 11 & 0,74 & 8 & 0,75 & 7 & 0,72 & 6 & 1,09 \\
\hline Enfermedad de Parkinson & 0 & 0 & 0 & 0 & 0 & 0 & 3 & 0,13 & 3 & 0,15 & 5 & 0,34 & 8 & 0,75 & 26 & 2,69 & 8 & 1,45 \\
\hline Epilepsia & 1 & 0,07 & 1 & 0,08 & 9 & 0,45 & 6 & 0,26 & 4 & 0,2 & 5 & 0,34 & 10 & 0,94 & 5 & 0,52 & 3 & 0,55 \\
\hline Inestabilidad y vértigo & 0 & 0 & 1 & 0,08 & 2 & 0,1 & 6 & 0,26 & 6 & 0,3 & 7 & 0,47 & 6 & 0,56 & 7 & 0,72 & 0 & 0 \\
\hline $\begin{array}{l}\text { Demencia vascular } \\
\text { y demencia mixta }\end{array}$ & 0 & 0 & 0 & 0 & 0 & 0 & 1 & 0,04 & 1 & 0,05 & 0 & 0 & 2 & 0,19 & 12 & 1,24 & 18 & 3,27 \\
\hline Temblor & 0 & 0 & 2 & 0,15 & 0 & 0 & 3 & 0,13 & 2 & 0,1 & 6 & 0,4 & 7 & 0,66 & 3 & 0,31 & 4 & 0,73 \\
\hline Epilepsia parcial & 1 & 0,07 & 3 & 0,23 & 6 & 0,3 & 4 & 0,17 & 5 & 0,25 & 4 & 0,27 & 4 & 0,38 & 2 & 0,21 & 1 & 0,18 \\
\hline Síndrome ansioso & 0 & 0 & 1 & 0,8 & 1 & 0,05 & 4 & 0,17 & 4 & 0,2 & 2 & 0,13 & 8 & 0,75 & 5 & 0,52 & 0 & 0 \\
\hline
\end{tabular}

Tabla III. Frecuencia absoluta y relativa de los diagnósticos registrados durante el período de estudio (2006-2007) clasificados por capítulos y categorías, según la CIE-10.

\begin{tabular}{|c|c|c|c|c|c|c|c|c|}
\hline & \multicolumn{2}{|c|}{ CIE-10 } & \multicolumn{2}{|c|}{2006} & \multicolumn{2}{|c|}{2007} & \multicolumn{2}{|c|}{ Total } \\
\hline & Capítulo & Categoría & $n$ & $\%$ & $n$ & $\%$ & $n$ & $\%$ \\
\hline Enfermedades episódicas y paroxísticas & 6 & G40-G47 & 152 & 27,4 & 117 & 22,3 & 269 & 25,1 \\
\hline Enfermedades degenerativas y desmielinizantes & 6 & G30-G37 & 100 & 18,1 & 100 & 19,1 & 200 & 18,6 \\
\hline Trastornos mentales orgánicos & 5 & F00-F09 & 70 & 12,6 & 88 & 16,8 & 158 & 14,7 \\
\hline Síndromes extrapiramidales & 6 & G20-G26 & 56 & 10,1 & 57 & 10,9 & 113 & 10,5 \\
\hline Enfermedades de la circulación cerebral & 8 & $160-169$ & 27 & 4,9 & 22 & 4,2 & 49 & 4,5 \\
\hline Trastornos relacionados con el estrés y somatomorfos & 5 & F40-F48 & 18 & 3,2 & 20 & 3,8 & 38 & 3,5 \\
\hline Enfermedades del oído interno & 7 & H80-H83 & 14 & 2,5 & 22 & 4,2 & 36 & 3,3 \\
\hline Enfermedades de la columna vertebral y de la espalda & 12 & M40-M54 & 8 & 1,4 & 18 & 3,4 & 26 & 2,4 \\
\hline Síntomas generales & 17 & R50-R69 & 13 & 2,3 & 9 & 1,7 & 22 & 2,0 \\
\hline Neuropatías y polineuropatías & 6 & G60-G64 & 15 & 2,7 & 7 & 1,3 & 22 & 2,0 \\
\hline Trastornos localizados de los nervios & 6 & G50-G59 & 16 & 2,9 & 3 & 0,6 & 19 & 1,8 \\
\hline Trastornos musculares y parálisis cerebral & 6 & $\begin{array}{l}\text { G70-G73 } \\
\text { G80-G83 }\end{array}$ & 8 & 1,4 & 10 & 1,9 & 18 & 1,7 \\
\hline Enfermedades de los tejidos blandos & 12 & M60-M79 & 8 & 1,4 & 10 & 1,9 & 18 & 1,7 \\
\hline Trastornos afectivos & 5 & F30-F39 & 12 & 2,2 & 5 & 1,0 & 17 & 1,6 \\
\hline Alteraciones visuales y ceguera & 7 & H54-H59 & 6 & 1,1 & 7 & 1,3 & 13 & 1,2 \\
\hline Otras & - & - & 31 & 5,6 & 29 & 5,4 & 60 & 5,5 \\
\hline Total & & & 554 & 100 & 524 & 100 & 1.078 & 100 \\
\hline
\end{tabular}

CIE-10: Clasificación Internacional de Enfermedades, 10. ${ }^{a}$ revisión. 
Tabla IV. Patología atendida estratificada por grupos diagnósticos (total de 2006 y 2007).

\begin{tabular}{lcc}
\hline & $n$ & $\%$ \\
\hline Trastornos cognitivos & 340 & 31,5 \\
\hline Cefaleas & 201 & 18,6 \\
\hline Trastornos del movimiento & 126 & 11,7 \\
\hline Enfermedades psiquiátricas & 71 & 6,6 \\
\hline Epilepsia & 70 & 6,5 \\
\hline Enfermedades cerebrovasculares & 57 & 5,3 \\
\hline Neuropatías & 54 & 5,1 \\
\hline Inestabilidad y vértigo & 36 & 3,3 \\
\hline Síncopes & 23 & 2,1 \\
\hline Enfermedades atróficas & 18 & 1,7 \\
\hline Otras enfermedades & 82 & 7,6 \\
\hline & 1.078 & 100 \\
\hline
\end{tabular}

III). El $61 \%$ de los diagnósticos correspondió a patologías del capítulo 6 -enfermedades del sistema nervioso central (G00-G99)-, y un 20,3\% al capítulo 5 -enfermedades mentales (F00-F99)-.

Por patologías, las más frecuentes fueron: trastornos cognitivos $(31,5 \%)$ -EA $(16 \%)$, deterioro cognitivo leve $(9,5 \%)$-, cefaleas $(18,6 \%)$-cefalea tensional $(9,7 \%)$, migraña $(7 \%)$-, trastornos del movimiento $(11,7 \%)$, enfermedades psiquiátricas $(6,6 \%)$, epilepsias $(6,5 \%)$, enfermedades cerebrovasculares $(5,3 \%)$ y neuropatías $(5,1 \%)$. Las demás no alcanzaron el $5 \%$ (Tabla IV).

Por frecuencia diagnóstica, el primer lugar lo ocuparon los trastornos cognitivos, en segundo lugar, las cefaleas, a las que siguieron las neuropatías $(5,3 \%)$, EP $(5,3 \%)$, epilepsia (3,9\%), inestabilidad y vértigo $(3,3 \%)$, demencia vascular y demencia mixta $(3,2 \%)$, y temblor $(3 \%)$. El resto de patologías no superó el 3\% (Tabla V). No hubo diferencias significativas en cuanto a la presentación anual en las enfermedades más prevalentes.

\section{DISCUSIÓN}

En nuestro estudio, la demanda asistencial de la atención neurológica de primeras consultas por 1.000 habitantes/año resultó muy reducida (4,3 para el año 2006 y 3,9 para el alo 2007) respecto a lo publicado por otros autores, que han hallado cifras que oscilan entre 7,6 [18] y 35 [28] casos por 1.000 habitantes. Bermejo [30], en una amplia revisión sobre la demanda asistencial en España y su futuro, advirtió que existe una gran variabilidad de la demanda y que las cifras más reducidas se observaron en un área rural de Cataluña y en otra en Bilbao. Nuestras diferencias podrían atribuirse, en gran medida, a que una parte de los pacientes, sobre todo los que padecen patologías más agudas, pueden haber sido atendidos en el Hospital Josep Trueta (de nivel III) que, a pesar de tener un área rural de referencia similar a la nuestra, dispone de un servicio de neurología con un mayor número de profesionales, guardias de la especialidad y un servicio de neuropediatría. De hecho, cuando suprimimos en nuestro estudio la población menor a 14 años, aumentamos el índice en casi dos puntos $(5,8)$.

Al igual que en la mayoría de los estudios, en nuestra consulta, la demanda asistencial se ha ido incrementando con la edad, con una clara preferencia por el sexo femenino [5,21,26$30]$, lo que posiblemente se relacione con la mayor esperanza de vida de las mujeres y la mayor prevalencia de algunas enfermedades neurodegenerativas, como la EA, en el sexo femenino. La mayoría de los autores atribuye a las cefaleas una mayor demanda asistencial de las mujeres, sobre todo por la mayor incidencia de las migrañas, aunque algunos autores opinan que simplemente se debe a una posible sobreutilización del sistema por parte de este colectivo [16,19,34,39]. De todos modos, las cefaleas, en los últimos 30 años, figuran, en la mayoría de los estudios, como la causa más frecuente de consulta neurológica, y así, ya en el año 1977 [43], en el que la edad media de los pacientes era de 41 años (2-78 años), el 56\% de las consultas provenía de mujeres, y la cefalea ( $32 \%$ de los casos) era la primera causa de consulta, lo que se ha mantenido en otros estudios posteriores [5,17-20,23,25,30,34,43]. Aunque las cefaleas generalmente se han mantenido como primera causa de consulta, a medida que la edad media de la demanda asistencial se ha incrementado, podemos observar cómo otras patologías, sobre todo las neurodegenerativas, se han ido incrementando, y así, en el área asistencial del Hospital Verge de la Cinta de Tortosa [17], en el que se analizó la evolución de la demanda asistencial en los años 1997 y 2003, observaron que en este período de tiempo la edad media de los pacientes atendidos pasó de 49,4 años (1997) a 56,7 años (2003), y aunque la patología diagnosticada más frecuente siguió siendo la cefalea $(23,2$ y $23,1 \%$, respectivamente), los trastornos cognitivos aumentaron del 7,2 al 16,7\%. Más recientemente, en el año 2006, se publicó otro artículo que hace referencia a la consulta neurológica ambulatoria del área de Rubí, en Barcelona [44], en el que observaron, igual que en nuestro trabajo, que la consulta más frecuente fueron los trastornos cognitivos (33\%) (demencia y deterioro cognitivo leve), seguidos de cefalea $(21 \%)$, trastornos del movimiento (9\%), enfermedades del sistema nervioso periférico $(2,5 \%)$, epilepsia $(6 \%)$ y enfermedad cerebrovascular (4,5\%). No cabe duda de que entre el trabajo de Sempere et al [34], realizado en el año 2000, uno de los estudios más amplios en España en población de más de 14 años de edad, en el que se observó como primer diagnóstico la migraña $(14,1 \%)$, seguida de cefalea tensional $(9,1 \%)$, epilepsia $(8,8 \%)$, EA $(5,7 \%)$, EP $(5,5 \%)$, síncope no neurológico $(3,7 \%)$, ansiedad $(3,5 \%)$, isquemia cerebral transitoria $(3,55)$, vértigo postural $(2,8 \%)$, trombosis cerebral $(2,7 \%)$ y temblor esencial (2,6\%); y en el de Rubí existen diferencias importantes en la distribución de los diagnósticos relacionada con la edad. Coincidimos en que la variabilidad de los diagnósticos depende en gran medida de la edad de los pacientes, y en que las enfermedades degenerativas cerebrales, vasculares y epilepsia son las más frecuentes en la población de más de 65 años.

Huerta-Villanueva [45] analiza y evalúa la demanda asistencial neurológica especializada ambulatoria de nuevas consultas entre los años 1997 y 2003 en el Hospital de Tortosa, que atiende a una población dispersa y rural, y en el Hospital de Viladecans, que atiende a un área más concentrada y metropolitana.

La codificación diagnóstica utilizada por Huerta-Villanueva [45] fue el CIE-9-MC, que define como un sistema de escasa precisión para el diagnóstico ambulatorio, y señala la necesidad de que el CIE-10-MC solucione este problema. Siguiendo estos criterios, en nuestro estudio reconvertimos los diagnósticos CIE-9-MC al CIE-10 y comprobamos que este nuevo sistema permite una agrupación de las categorías diagnósticas más acorde con los diagnósticos que realizamos en la práctica clínica habitual. Observamos que los diagnósticos neurológicos, teniendo en cuenta el encuadre nosológico de los pacientes, se agruparon 
un $61 \%$ en el capítulo 6 , que hace referencia a las enfermedades del sistema nervioso central y de los órganos sensoriales, y un $20,3 \%$ en el capítulo 5, que hace referencia a los trastornos mentales. Esta agrupación demuestra que los neurólogos atienden aproximadamente, de cada tres pacientes, a uno con patología psiquiátrica.

Con el fin de comparar nuestros resultados con los de la tesis de Huerta-Villanueva, ajustamos nuestra población a algunas variables analizadas por él, comprobando que la frecuentación de las enfermedades atendidas por los neurólogos de nuestro servicio se mantiene estable hasta los 50 años y progresa de modo significativo a partir de esta edad. Nuestros datos difieren de los suyos en que la frecuentación en nuestra población sigue progresando después de los 79 años, posiblemente porque está más envejecida.

En cuanto a la patología por grupos diagnósticos, observamos una clara diferencia entre la expuesta por Huerta-Villanueva [45], en la que el diagnóstico más frecuente fue 'cefalea', y nuestro estudio, en el que es el 'trastorno cognitivo'. Esta diferencia podría justificarse por el especial interés y dedicación que tienen los médicos de nuestra institución por las enfermedades mentales, puesto que el Institut d'Assistència Sanitària es el centro de referencia para las enfermedades psiquiátricas de toda la provincia, y por el hecho de que el Servicio de Neurociencias Clínicas incluye la UVaMiD. Coincidimos con Huerta-Villanueva en que los trastornos del movimiento, la epilepsia, las patologías psiquiátricas y las enfermedades cerebrovasculares son enfermedades de alta prevalencia.

En cuanto a los diagnósticos genéricos más frecuentes, en nuestro estudio se observa que el 16,1\% de los pacientes atendidos fueron diagnosticados de EA, lo que duplica esta enfermedad respecto a la serie de Huerta-Villanueva [45], y es la primera causa de diagnóstico en nuestro servicio. Destacamos también que el deterioro cognitivo leve corresponde a un 9,5\% de los pacientes atendidos, lo que indica el interés de los médicos de nuestras áreas básicas de referencia en el estudio de los trastornos cognitivos y/o la demencia.

La cefalea tensional, la migraña, las neuropatías, la EP y la epilepsia son un grupo de enfermedades que, al igual que en la serie de Huerta-Villanueva, son frecuentes en nuestras consultas. Nuestros resultados están más cerca de los que publicaron Fragoso y Aguilar-Barberá [44], lo que pone de manifiesto que la demanda de visitas neurológicas por parte de los especialistas en medicina primaria se está modificando en relación con que la mayor demanda de consultas se realiza basándose en las enfermedades neurodegenerativas propias de la edades avanzadas, lo que queda claramente reflejado en el IF de la EA, deterioro cognitivo leve, EP, demencia vascular y demencia mixta, que aumentan considerablemente a partir de los 60 años.

En resumen, los resultados de nuestro estudio y la revisión bibliográfica sugieren que, en nuestro país, en los últimos 30 años, existió una relativa variabilidad en la demanda de las visitas especializadas por parte de los médicos de atención primaria, y que ésta se ha ido modificando en relación con el envejecimiento poblacional, observándose un incremento de las patologías neurodegenerativas. En la actualidad, y de modo general, pueden distinguirse dos grupos de patologías neurológicas según el grado de frecuentación: por un parte, las enfermedades episódicas y paroxísticas, las enfermedades degenerativas y desmielinizantes, los trastornos psicóticos y del comportamiento y los síndromes extrapiramidales, que presentan, en general, una
Tabla V. Diagnósticos más frecuentes de los pacientes de nuestra zona de referencia (> 1\%) (total de 2006 y 2007 ).

\begin{tabular}{|c|c|c|}
\hline & $n$ & $\%$ \\
\hline Enfermedad de Alzheimer & 172 & 16,0 \\
\hline Cefalea tensional & 105 & 9,7 \\
\hline Deterioro cognitivo leve & 102 & 9,5 \\
\hline Migraña & 75 & 7,0 \\
\hline Neuropatías & 57 & 5,3 \\
\hline Enfermedad de Parkinson & 57 & 5,3 \\
\hline Epilepsia & 42 & 3,9 \\
\hline Inestabilidad y vértigo & 36 & 3,3 \\
\hline Demencia vascular y demencia mixta & 34 & 3,2 \\
\hline Temblor & 32 & 3,0 \\
\hline Epilepsia parcial & 29 & 2,7 \\
\hline Síndrome ansioso & 28 & 2,6 \\
\hline Síncope & 23 & 2,1 \\
\hline Otros trastornos del movimiento & 22 & 2,0 \\
\hline Trombosis cerebral & 21 & 1,9 \\
\hline Síndrome depresivo & 20 & 1,9 \\
\hline Accidente isquémico transitorio & 19 & 1,7 \\
\hline Neuralgia del trigémino & 18 & 1,7 \\
\hline Enfermedades atróficas & 18 & 1,7 \\
\hline Demencia por cuerpos de Lewy & 14 & 1,3 \\
\hline Otras demencias degenerativas & 14 & 1,3 \\
\hline Otros trastornos psiquiátricos & 11 & 1,0 \\
\hline Otras enfermedades & 64 & 5,9 \\
\hline Diagnósticos inferiores al 1\% & 65 & 6,0 \\
\hline Total & 1.078 & 100 \\
\hline
\end{tabular}

elevada frecuentación; y, por otra, las enfermedades con moderada o baja frecuentación, como las enfermedades cerebrovasculares, los trastornos fóbicos y neuróticos, las enfermedades del oído interno, las enfermedades de la columna vertebral y de la espalda, los síntomas neurológicos generales, y las neuropatías y las polineuropatías.

Desde otro punto de vista, el de la edad, distinguimos dos tipos de demandas: las de la población más joven, que consulta por cefalea, migraña y crisis epilépticas, y las de edad más avanzada, que consulta por trastornos extrapiramidales y enfermedades degenerativas y desmielinizantes.

Otro grupo de patologías, de baja frecuentación, pueden considerarse enfermedades de edades intermedias, como son los trastornos psicóticos y del comportamiento, las enfermedades del oído interno o los trastornos afectivos, que no son en sentido estricto patologías que requieran específicamente la vista en un servicio de neurología, sino que pueden ser atendidas por otros dispositivos sanitarios, aunque son patologías que entran en el 
diagnóstico diferencial con enfermedades orgánicas cerebrales, lo que justificaría la consulta, pero no el seguimiento.

Un aspecto importante que se debe considerar es la relación de la frecuentación y la discapacidad o dependencia que pueda generar la patología específica. Por ejemplo, a pesar de que las enfermedades episódicas y paroxísticas tienen una elevada frecuentación, en términos de discapacidad o dependencia asociada su impacto es bajo. Sin embargo, las enfermedades degenerativas y desmielinizantes y los síndromes extrapiramidales sí generan en el paciente un progresivo deterioro cognitivo y/o funcional que provoca dependencia. En este sentido, los resultados de este trabajo y de los estudios previos muestran un incremento de la frecuentación con el aumento de la edad.

Finalmente, señalamos que la promoción de una correcta clasificación diagnóstica de las patologías neurológicas en la consulta ambulatoria es fundamental para poder disponer de información relevante y representativa de su frecuentación en el territorio y de las necesidades actuales y futuras de los dispositivos neurológicos. Además, se trata de información de una gran utilidad para la planificación y distribución de los recursos requeridos por la Ley de la dependencia para todas las patologías que provocan discapacidad. En este sentido, la clasificación propuesta por la CIE-10-MC parece más adecuada que la codificación CIE-9-MC, que fue desarrollada fundamentalmente para la codificación de diagnósticos y procedimientos de pacientes en el alta hospitalaria y es un sistema de codificación con una baja precisión para el diagnóstico de la patología ambulatoria. En conclusión, la utilización de este sistema de codificación, ya utilizado en la atención primaria de nuestra zona, debe promoverse en las unidades ambulatorias de neurología de nuestro territorio, con la finalidad de que en los próximos años médicos de familia y neurólogos hablemos un mismo lenguaje y podamos analizar y compartir nuestras experiencias para adecuar los recursos sociosanitarios a las necesidades de nuestra población.

\section{BIBLIOGRAFÍA}

1. Population Division of the Department of Economic and Social Affaire of the United Nations Secretariat. World Population Prospects. The 2004 Revision and World Urbanization Prospect. The 2006 Revision. URL: http://www.un.org/esa/population. [01.02.2008].

2. MacDonald BK, Cockerell OC, Sander JW, Shorvon SD. The incidence and lifetime prevalence of neurological disorders in a prospective community-based study in the UK. Brain 2000; 123: 665-76.

3. Casabella B, Aguilar M, Jauma, S. La patología neurológica extrahospitalaria: una visión clinicoasistencial de la demanda atendida por el neuropsiquiatra y el médico general. Rev Neurol 1990; 18: 197-202.

4. Murray CJL, López AD. The global burden of disease. World Health Organization and the World Bank. Cambridge: Harvard School of Public Health; 1996

5. Pondal M, Bermejo F, Del Ser T. La asistencia neurológica ambulatoria extrahospitalaria. Análisis de la demanda registrada durante 18 meses en una consulta jerarquizada de neurología. Neurologia 1989; 4: $124-31$

6. Sicras-Mainar A, Velasco-Velasco S. Patrón de uso de recursos y costes en pacientes que demandan atención por trastornos neurológicos en atención primaria. Rev Neurol 2007; 45: 641-6.

7. Gascon-Bayarri J, Reñé R, Del Barrio JL, De Pedro J, Ramón JM, Manubens JM, et al. Prevalence of dementia subtypes in El Prat de Llobregat, Catalonia, Spain: the PRACTICON study. Neuroepidemiology 2007; 28: 224-34.

8. Vilalta-Franch J, López-Pousa S, Llinàs-Reglà J. Prevalencia de demencias en una zona rural. Estudio de Girona. Rev Neurol 2000; 30: 1026-32.

9. Clavería LE, Duarte J, Sevillano MD, Pérez-Sempere A, Cabezas C, Rodríguez F, et al. Prevalence of Parkinson's disease in Cantalejo, Spain. A door-to-door survey. Mov Disord 2002; 17: 242-9.

10. Benito-León J, Bermejo-Pareja F, Rodríguez J, Molina JA, Gabriel R, Morales JM. Neurological Disorders in Central Spain (NEDICES) Study Group. Prevalence of PD another types of parkinsonism in three elderly populations of central Spain. Mov Disord 2003; 18: 267-74

11. Crisp AH, Kalucy RS, McGuinness B, Ralph PC, Harris G. Some clinical, social and psychological characteristics of migraine subjects in the general population. Postgrad Med J 1977; 53: 691-7.

12. Castillo J, Muñoz P, Guitera V, Pascual J. Epidemiology of chronic daily headache in the general population. Headache 1999; 39: 190-6.

13. Artigas J, Grau R, Canosa P, Esclusa F. Prevalence and characteristic of infantile headache in selected area. Cephalalgia 1997; 17: 293.

14. White KL. Fundamentos y práctica de la planificación y de la gestión nacional de la salud. Cuadernos de Salud Pública n. ${ }^{\circ}$ 46. Ginebra: Organización Mundial de la Salud; 1977.

15. Martínez-Salio A, Oliet C, Porta-Etessam J, Bermejo-Pareja F. Análisis de la demanda asistencial de la población inmigrante en una consulta de neurología extrahospitalaria. Neurologia 2003; 18: 425-30.

16. González-Menacho J, Olivé-Plana JM. Epidemiología de la patología neurológica ambulatoria en el Baix Camp (Tarragona) durante 1999. Neurologia 2001; 16: 154-62.

17. Huerta-Villanueva M, Baiges-Octavio JJ, Martín-Ozaeta G, MuñozFarjas E, Rubio-Borrego F. Evolución de la demanda de asistencia neurológica ambulatoria y patología atendida en la consulta de neu- rología de la región sanitaria de Tortosa, Tarragona. Rev Neurol 2005; 41: 68-74.

18. Batalla X. Neurología extrahospitalaria. Análisis descriptivo de una consulta en el Baix Llobregat. Rev Neurol 1997; 25: 1546-50.

19. López-Domínguez JM, Rojas-Marcos I, Sanz-Fernández G, BlancoOllero A, Robledo-Strauss A, Díaz-Espejo C. Análisis descriptivo de la demanda de asistencia neurológica ambulatoria en una área de Huelva. Rev Neurol 2007; 44: 527-30.

20. López-Hernández N, Espinosa-Martínez J. Análisis descriptivo de la asistencia neurológica ambulatoria en Elche, Alicante. Rev Neurol 2007; 45: $219-23$.

21. Escudero J, Cano M, Martínez-Moreno I. Estudio comparativo entre la asistencia neurológica extrahospitalaria con el resto de especialidades médicas en el distrito sanitario de Xàtiva. Rev Neurol 1994; 22 : 432-4.

22. Muñoz R. Asistencia neurológica extrahospitalaria. Estudio prospectivo de las zonas de salud del área 13; centro de especialidades de Sueca (1992-1993). Rev Neurol 1994; 22: 435-6.

23. Baquero M, Domínguez FJ, Vílchez JJ, Sevilla T, Casanova B. La asistencia médica en neurología: perspectiva actual desde los datos iniciales de una consulta extrahospitalaria. Rev Neurol 1994; 22: 427-31.

24. Catalá J. La asistencia neurológica en los hospitales del área y centros de especialidad de la Comunidad Valenciana en 1994. Rev Neurol 1995; 23: 1111-5.

25. Antigüedad A, Bárcena J, Pérez-Bas M, Aldape J, Hurtado P, Zarranz JJ. Actividad asistencial de los facultativos especialistas en el área de neurología. Experiencia en Vizcaya. Neurologia 1995; 10: 324-9.

26. Gracia-Naya M, Marta E, Usón M, Carod J. Estudio epidemiológico descriptivo de una consulta externa de neurología. Rev Neurol 1996; 24: 633-7.

27. Villagra P, Cubero A. Estudio de la demanda asistencial en una consulta jerarquizada de neurología correspondiente al área norte de Las Palmas de Gran Canaria. Rev Neurol 1997; 25: 1544-6.

28. Morera-Guitart J, Comisión de Análisis de la Calidad de la Sociedad Valenciana de Neurología. Estudio transversal multicéntrico de la asistencia neurológica extrahospitalaria de la Comunidad Valenciana. Rev Neurol 1998; 27: 438-6.

29. Bermejo F, Calandre L, Morales MA, Gutiérrez-Rivas E, Mateos F, Molina JA, et al. Estimación de la demanda neurológica en un área sanitaria de Madrid (área 11, Hospital Universitario 12 de Octubre). Neurologia 1999; 14: 444-51.

30. Bermejo F. Demanda neurológica en España. Datos para un futuro más exigente. Rev Neurol 1999; 29: 673-7.

31. García-Albea E. El devenir de la neurología y de la asistencia neurológica en el marco público español. Neurologia 2000; 15: 32-5.

32. Herrera M, Gracia R, Santana R, Jiménez A, Ayala J, Cuadrado P. Demanda asistencial neuropediátrica en un hospital general. An Esp Pediatr 2000; 53: 106-11.

33. Junta Directiva y Comité Asesor de la SEN. Presente y futuro de la neurología española. Neurologia 2001; 16: 408-17.

34. Sempere AP, Mola S, Medrano V, Esguevillas T, Costa C, Salazar V, et al. Epidemiología descriptiva de la asistencia neurológica ambulatoria en el área de la Vega Baja (Alicante). Rev Neurol 2002; 35: 822-6. 
35. Morera-Guitart J, Pedro-Cano MJ. Variación en la patología atendida en las consultas de neurología: un futuro demencial. Neurologia 2003; 18: 417-24.

36. Olivé JM, González-Menacho J, González-Menacho S. Calidad de las derivaciones a consulta ambulatoria de Neurología. Neurologia 2003; 18: 499.

37. Aranaz JM, Mira JJ, Beltrán J. La gestión por procesos asistenciales integrados. Neurologia 2003; 18: 48-56.

38. Villarejo A, Porta-Etessam J, Martínez-Salio A, De Frutos V, Ruiz J, Almajano J, et al. Análisis comparativo de la asistencia neurológica ambulatoria en el área sanitaria 11 de Madrid entre los años 1986-1987 y 2004. Neurologia 2004; 19: 647.

39. Pérez-Carmona N, Sánchez-Pérez RM, Abellán-Miralles I, Díaz-Marín C. Asistencia neurológica ambulatoria en el área de la Marina Baixa, Alicante. Rev Neurol 2004; 39: 607-13.

40. Morera-Guitart J. Asistencia neurológica ambulatoria en España. Pasado, presente, ¿futuro? Rev Neurol 2005; 41: 65-7.

41. Lacruz L, Escudero J, Parra J, Brocalero A, Castillo S. Registro de la actividad asistencial neurológica ambulatoria del área 9. Rev Neurol 2006; 42: 622 .
42. López-Pousa S. El servicio de neurología dentro de la estructura hospitalaria: dimensión social. Anales del Hospital de la Santa Cruz y San Pablo 1977; 5: 211-6.

43. López M. Hospital outpatients clinics, a neurology audit in South Catalonia. J Neurol Neurosurg Psychiatry 1992; 55: 522.

44. Fragoso M, Aguilar-Barberá M. Análisis de una consulta de neurología ambulatoria en el área de Rubí, Barcelona. Rev Neurol 2006; 42: 58-9.

45. Huerta-Villanueva M. Análisis de la asistencia neurológica en dos hospitales comarcales de las áreas de las Terres de l'Ebre y Baix Llobregat [tesis doctoral]. Barcelona: Universitat de Barcelona; 2006.

46. National Center for Health Statistics. International Classification of Diseases. Ninth Revision-Clinical Modification. URL: http://www.cdc. gov/nchs/icd9.htm. [25.10.2008].

47. National Center for Health Statistics. International Classification of Diseases, Tenth Revision-Clinical Modification. URL: http://www.cdc. gov/nchs/about/otheract/icd9/abticd10.htm. [25.10.2008].

48. Departament de Salut, Generalitat de Catalunya. Classificació Internacional de Malalties. 9a revisió. Modificació clínica. Barcelona: Proa; 1997.

49. Instituto Nacional de Estadística. URL: http://www.ine.es. [25.10.2008].

\section{ANALYSIS OF THE DEMAND FOR NEUROLOGICAL HEALTH CARE GENERATED BY PRIMARY CARE IN A GEOGRAPHICAL AREA WITHIN THE DISTRICTS OF GIRONA}

Summary. Aim. To perform a descriptive analysis of the outpatient activity in a neurological department in terms of the frequency and type of neurological diseases that were attended. Patients and methods. A retrospective and cross-sectional study was conducted involving patients referred to the neurology outpatients department. The cases that visited for the first time during the years 2006 and 2007 were recorded consecutively. Medical information was evaluated based on computerised hospital registers and the following data were collected: health referral area, date of visit, age, sex and diagnosis according to the International Classification of Diseases, 9th edition, clinical modification (CIE-9-MC), reconverted into the International Classification of Diseases, 10th edition (CIE-10). Results. The mean age was $60.6 \pm 20.9$ years (range: $4-95$ years) and $61.4 \%$ were females. The rate of frequency of visits was 4.3 requests/1000 inhabitants for the year 2006 and 3.9 for the year 2007; an increase was observed with age for the neurodegenerative diseases. As far as the CIE-10 is concerned, the diseases were classified as episodic and paroxysmal (25\%), degenerative and demyelinating (18.6\%), organic mental disorders (14.7\%), extrapyramidal syndromes (10.5\%), diseases affecting cerebral circulation (3.5\%), stress-related disorders and somatomorphs (3.5\%) and diseases of the inner ear (3.3\%). The remaining pathologies did not reach $3 \%$ of the total. Diseases of the central nervous system were observed in $61 \%$ of the patients and psychiatric disorders were found in $20.3 \%$. The most common diseases were cognitive disorders (31.5\%), headaches (18.6\%) and movement disorders (11.7\%), followed by psychiatric diseases, epilepsies, cerebrovascular diseases and neuropathies. Conclusions. The frequency of visits increases with age and the order, in terms of frequency, was: episodic and paroxysmal, degenerative and demyelinating, psychotic and behavioural disorders, and extrapyramidal syndromes. [REV NEUROL 2009; 49: 288-94]

Key words. Diagnosis. Epidemiology. Neurological demand. Neurological diseases. Primary health care. 